\title{
Twenty years of confined colloids: from confinement-induced freezing to giant breathing
}

\author{
H Löwen \\ Institut für Theoretische Physik II: Weiche Materie, Heinrich-Heine-Universität \\ Düsseldorf, Universitätsstraße 1, D-40225 Düsseldorf, Germany \\ E-mail: hlowen@thphy.uni-duesseldorf.de
}

\begin{abstract}
The physics of colloidal suspensions confined in slits and cavities has significantly advanced during the last twenty years. In particular, freezing transitions in confinement have been addressed by theory and simulations and experimental realizations were proposed to confine colloidal particles to two dimensions. After reviewing this progress, we discuss the generalization to time-dependent confinement which leads to nonequilibrium situations. This is elaborated further for unstable situations where the particles can leave the confinement. In particular, the completely overdamped Brownian motion of a colloidal particle in a time-dependent harmonic trap is considered. The analytically soluble model of a time-dependent quadratic potential is used to extract the dynamical properties of the potential if the potential undergoes periodic switching from a confining harmonic potential to an unstable one. The amplitudes of the oscillating particle response can strongly grow in time which we refer to as "giant breathing". This giant breathing process occurs also in anharmonic potentials and is verifiable in real-space experiments of colloids in laser-optical fields.
\end{abstract}

PACS numbers: 82.70.Dd, 05.40.Jc, 


\section{Introduction}

Confined fluids in slit-like geometries exhibit structural [1] and dynamical [2] properties which are vastly different from their three-dimensional bulk counterparts. For example the crystallization transition may change from a first-order transition in the threedimensional bulk to a two-stage continuous transition in $2 \mathrm{D}$ with an intermediate hexatic phase possessing long-ranged bond-orientational order, the latter situation is referred to as the Kosterlitz-Thouless-Nelson-Halperin-Young scenario [3]. Mesoscopic colloidal suspensions represent ideal model systems [4] to study two-dimensional systems as they can be confined in a controlled way down to two-dimensions and are therefore ideal realizations of classical two-dimensional systems.

In the past twenty years, considerable progress has been achieved to understand confined colloidal suspensions under various conditions and for different physical questions. The goal of this paper is twofold: first of all, we shall briefly review the basic progress obtained in the physics of confined colloidal suspensions in the past two decades where we focus in particular on crystallization and particle dynamics in confinement. There we discuss step by step: i) charged colloids between charged glass plates, ii) superparamagnetic colloids exposed to an external magnetic field which are confined to an air-water interface, and iii) colloids in static laser-optical gratings and traps. Then we generalize the situation towards a time-dependent confinement as e.g. for colloids in time-dependent external optical traps. Second, we consider the situation of a time-dependent confinement which is unstable, i.e. where the particles are allowed to escape. Though in principle experimentally accessible, this problem has received little attention for colloids so far. Here we consider the simplest case of a single particle in a trap which switches periodically in time from stable to unstable confinement.

As a result we find a giant breathing mode of the particle which moves in a timedependent oscillating harmonic potential switching from a stable to unstable situation. The amplitude of the particle's mean-square displacement from the origin are amplified exponentially in time. Optical traps can by now be prepared in reality almost in an arbitrary way [5] by a suitable interference of several laser beams. Therefore the giant breathing effect can in principle be verified in microscopy experiments of trapped colloidal particles.

The paper is organized as follows: chapter II will highlight some progress in our understanding of confined colloids during the last decades. We shall focus on charged suspensions between glass plates, on superparamagnetic suspensions pending at an airwater interface and on particles in external laser-optical fields. In chapter III, we shall describe the new phenomenon of giant-breathing in terms of a simple model for a colloid moving by Brownian dynamics in an unstable laser-optical trap. We conclude and give a list of possible future research topics in chapter IV. 


\section{Confined colloidal suspensions: progress during the last twenty years}

\subsection{Charged suspensions confined between glass plates}

The basic idea originating from the years 1980-1990 is to confine mesoscopic charged colloidal particles (e.g. polystyrene spheres) in aqueous solution between two parallel glass plates of micrometer spacing $[6,7,8,9,10]$. This realizes an extreme confinement to a slit where the thickness is comparable to the particle size. A corresponding confinement is obviously much harder to do in molecular or atomic systems. The glass plates get charged in water, typically with the same sign of charge as the colloids such that the particle-wall interacting is repulsive. An actual estimate of the repulsion strength $[11,12]$ shows that it can be considerably larger than the thermal energy $k_{B} T$. Therefore the system is confined almost completely to the middle of the slit and out-of-plane fluctuations can be held small. The second advantage is that videomicroscopy is applicable to observe the system through the glass plates in order to follow the individual particle trajectories. These trajectories are Brownian due to the random kicks with the solvent but also dominated by the colloid-colloid interaction. Finally, the third advantage is that the interparticle repulsion can be continuously tuned by the concentration of added salt.

In a pioneering work of Murray et al, clear indications for the Kosterlitz-ThoulessNelson-Halperin-Young scenario of two-dimensional melting were found for a colloidal monolayer $[13,14]$. Still some statistical inaccuracies were remaining as the identification of the intermediate hexatic phases requires the knowledge about the long-distance behaviour of bond-orientational correlations.

The realization of charged colloids between charged plates opens further the fascinating possibility to tune the wall repulsion (or the particle density) in order to move from monolayers to bi and multilayers. If the two glass plates are not exactly parallel forming a wedge, one can follow a whole cascade of solid-to-solid transition along the wedge as shown in figure 1.

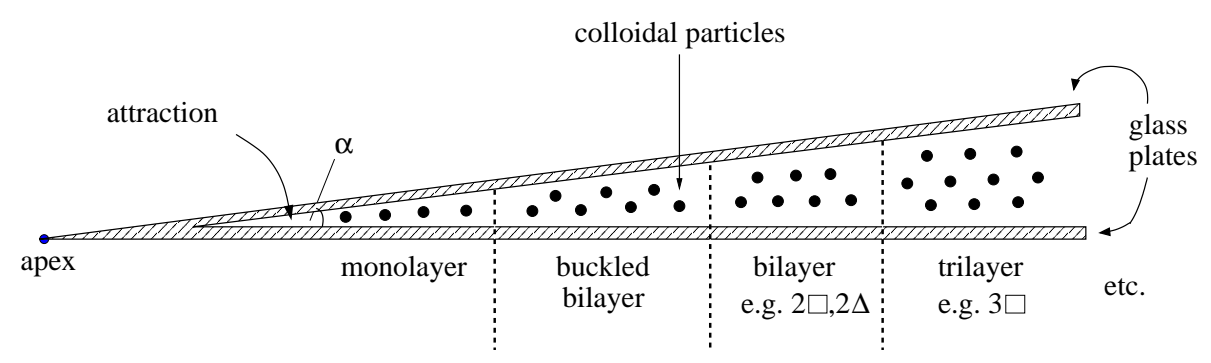

Figure 1. Sketch of a wedge confinement of colloidal particles. For small opening angles $\alpha$, there is a cascade of multilayered crystalline phases as a function of distance to the apex of the wedge. The attraction close to the wedge apex is also indicated.

The details of the cascade have been debated during the last decades. Regarding theory, the simplest model is that of hard spheres between hard walls. The close- 
packing structure reveals a cascade of solid-to-solid transformations if the wall distance is increasing [15]. The simplest cascade here is

$$
n \Delta \rightarrow(n+1) \square \rightarrow(n+1) \Delta, \quad n=1,2,3, \ldots
$$

starting with a triangular monolayer. This picture is too simplistic, however, since already during the $1 \Delta \rightarrow 2 \square$ transition a buckled bilayer phase is intervening $[15,16]$ corresponding to a staggered crystal with two intersecting rectangular lattices. These continuously transform into a $2 \square$ bilayered crystal consisting of two staggered square lattices. Upon further increasing the plate distance, the close-packed configuration is getting rhombic $[17,18]$ until a bilayer structure with two intersecting triangular crystals is reached $(2 \Delta)$. Beyond the bilayer regime which is limited by $2 \Delta$, the cascade is even more complicated [19]:

$$
2 \Delta \rightarrow 2 \text { hcp-like } \rightarrow \text { 2hcp }(100) \rightarrow 2 \text { hcp-like } \rightarrow 2 P_{\square} \rightarrow 3 \square
$$

and involves a prism phase $\left(P_{\square}\right)$ and more complicated cut-outs and derivations from the $h c p$ phase $[20,21,22]$. All of the corresponding structures have been confirmed in the experiment in the limit of high salt concentration [20, 21, 23].

For charged suspensions, a Yukawa pairwise effective interaction [24, 25, 26, 27] is more realistic than those of hard spheres. This stays to be a reasonable model if the colloids are confined between glass plates $[11,12]$. The crystalline layering scenario was explored by lattice-sum minimizations at zero temperature [28] and two different rhombic situations where found depending on the screening strength. The rhombic structures have a different basis, i.e. there are no isostructural transitions [29]. The bilayer crystals were confirmed in experimental studies on charged suspensions [30]. Finally, the crossover to higher-order multilayers is even more intriguing involving more complicated intermediate structures than the hard-sphere cascade (2) [31, 32, 33, 19].

Finally, there is a striking attraction for the colloids in the cusp of the wedge. This effective attraction can be attributed to the plate-particle repulsion. The effect is obtainable from linear screening theory and from computer simulations with explicit microions and might explain the experimental fact that colloidal particles stick in the corners and cusps [34]. This is indicated in figure 1 as well.

Another important topic for colloids under confinement is the shift of the kinetic glass transition relative to the bulk glass transition. It has been shown by using computer simulations of various models $[35,36]$ that the sign of the shift depends on details of the wall-particle interactions. Real-space experiments on colloidal dispersions have also revealed a subtle dependence on gravity [37]. For smooth confining and repulsive walls, both simulations [38] and experiments [39] show that the glass transition in confinement shifts to lower densities as compared to the bulk.

\subsection{Superparamagnetic suspensions confined to an interface}

By using the response of colloids to a magnetic field, almost perfect two-dimensional confinement can be achieved $[40,41,42]$. The idea here is to consider a pending water 
droplet at a glass plate which is filled with superparamagnetic particles. Due to gravity the particle sink down until they meet the air-water interface. Since the surface tension of the air-water interface is high, the particles do not penetrate through the interface as this would require additional interfacial area. Consequently the particles are confined to the interface by combination of gravity acting downwards and interfacial free energy keeping them upwards. Since the water droplet containing the colloidal particles is macroscopic, its air-water interface is flat on a micron scale typical for an interparticle spacing between the colloids. Now an external magnetic field $\vec{B}$ is applied which induces magnetic dipole moments in the particles, $\vec{m}=\chi \vec{B}$. This in turn results in a dipoledipole interaction between the particles. All dipole moments are along the magnetic field direction. If the external magnetic field is perpendicular to the interface, there is a repulsive interaction between the particles which can be described by a pairwise potential which scales as the inverse cube of the distance [43]. The prefactor scales with the square of the magnetic dipole moment, i.e. with the square of the magnetic field strength $\vec{B}^{2}$. By tuning the strength of $\vec{B}$, one can thus readily change the interaction strength. For inverse power-law interactions, this corresponds formally to a change of temperature or density. The experimental set-up is combined with video-microscopy in order to visualize the individual particle trajectories.

Recent progress has been achieved for one-component and two-component systems which is outlined as follows: For one-component systems, the Kosterlitz-ThoulessNelson-Halperin-Young scenario was unambiguously confirmed on this strictly twodimensional system [44]. Moreover, it was confirmed that Young's modulus approaches $16 \pi$ at the melting temperature [45] as predicted by the theory. The modulus of orientational stiffness was measured in the hexatic phase at the fluid-hexatic transition and found to be $76 / \pi[46]$, again in agreement with theory. The creation of disclinations and dislocations is crucial for the melting scenario, the pair interaction of dislocations has been determined in these two-dimensional crystals [47]. Finally, the crystal phonon dispersion relations [48] have been determined and found in full quantitative agreement with theoretical calculations [49].

If the external magnetic is tilted relative to the surface normal, anisotropic dipoledipole interactions between the particles result. The zero-temperature phase diagram was calculated by lattice sums [43] revealing a wealth of anisotropic stable solid lattice in agreement with experimental data [50]. The Lindemann parameters in the anisotropic crystals were determined in good agreement between experiment and theory [51]. The melting of the anisotropic crystals is again mediated by defects [52] as in the isotropic case, the resulting intermediate phase can be called "smectic-like".

Two-component (binary) systems with big and small magnetic dipole moments represent ideal glass formers in two spatial dimensions [53]. Several structural and dynamical features of these mixtures have been explored including the long-time self diffusion [54] and the partial clustering of the small particles at moderate interaction strengths [55]. The latter is revealed by an unusual peak in the partial structure factor of the small-small pair correlations. By suddenly increasing the magnetic field, the system 
can be quenched on a time scale which is much smaller than the particle diffusion [56]. Since magnetic field strength corresponds formally to temperature, an ultrafast temperature quench can be realized experimentally which is very difficult for molecular systems.

Analyzing the particle configuration after a rapid quench reveals some local crystalline patches in the glass $[57,58]$. These patches correspond to the thermodynamically bulk crystal [59] revealing an interplay between vitrification and crystallization. At present, the associated dynamical heterogeneity in the glass is explored. A simulation snapshot of a rapidly quenched binary system is shown in figure 2. The colored particles indicate region with local crystalline order (either triangular and quadratic) and are clearly highlighting the crystalline patches.

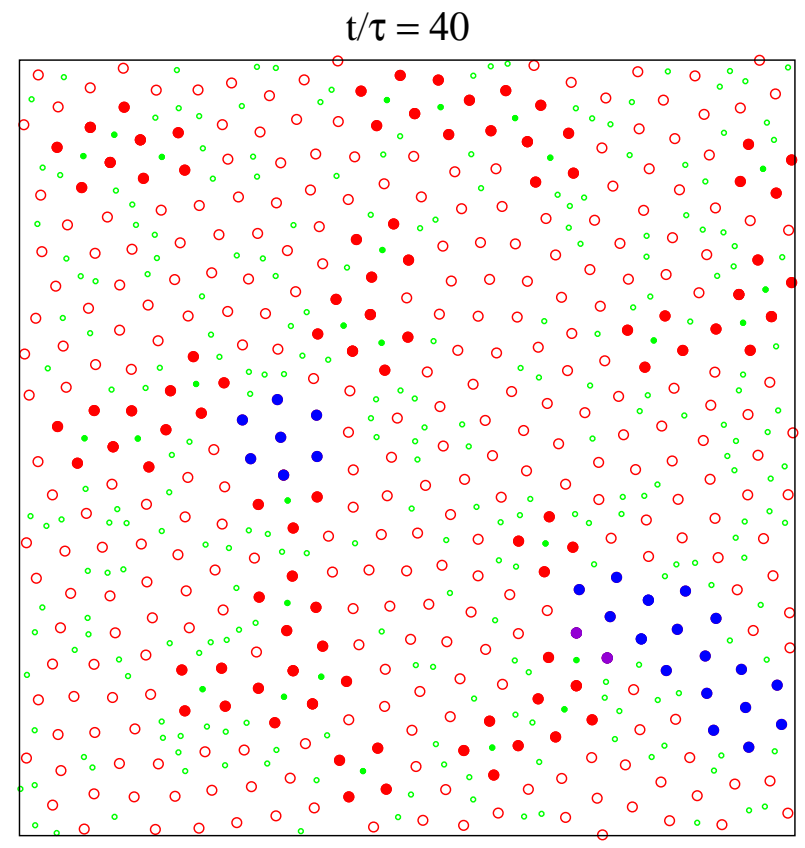

Figure 2. Brownian dynamics computer simulation snapshot of a two-dimensional binary system of parallel dipoles which is quenched instantaneously from an almost non-interacting system to strong interaction by increasing the external magnetic field strength. The time after the quench is $t / \tau=40$ with $\tau$ denoting the time a big particles diffuses over its own diameter. The ratio of the dipole moments is 0.1 and the relative composition of the small particles is $X=0.4$. Crystalline patches are identified resulting in colored particles. A blue colored big particle belongs to a crystalline triangular surrounding and a red colored particle belongs to a square surrounding. All other big particles are shown in circled red color. Few big particles belonging to both triangular and square surroundings are shown in pink color. The small particles are shown in green if they belong to a square center of big particles, otherwise they appear in circled green. 


\subsection{Colloidal particles in static laser-optical fields and traps}

A static external potential which is periodic in one spatial direction can be realized by crossing laser beams and using the dielectric contrast of colloidal particles relative to the solvent as a source of attraction into the regions of high laser intensity. In simplest form, the corresponding external potential felt by the colloids reads

$$
V(z)=V_{0} \cos (k x),
$$

where $V_{0}$ is an energy amplitude and $k$ is the wave number of the optical grating. The freezing behaviour of colloidal particles in this "substrate" potential has been explored to about more than twenty years ago by Ackerson and coworkers leading to the scenario of laser-induced freezing [60] which exhibits interesting reentrant effects [61, 62, 63]: as the prefactor $V_{0}$ is continuously increasing, the system undergoes first a fluid-solid and then again a solid-fluid transition. The corresponding situation of a liquid-gas transition under the potential (3) was also investigated in the context of colloid-polymer mixtures and a split of the bulk critical point into two critical points separated by an intermediate structured liquid was found [64].

Much related work has been done for colloids in parabolic traps which is the simplest model for optical tweezers

$$
V(r)=\frac{1}{2} V_{0} r^{2} .
$$

Filling colloidal particles into a harmonic trap results typically in a shell structure with magic numbers (see, e.g. [65, 66, 67, 68]) where interesting dynamical behaviour is observed as well.

Gratings which are periodic in space in two spatial directions have also been considered and studied. In particular, underlying triangular lattices will lead to molecular crystals where a "molecule" consists of a cluster of colloids all of which assemble in the same well (forming e.g. trimers) [69, 70]. These molecular crystals show a melting and freezing behaviour which is drastically different from bulk crystals of spherical particles. A two-step melting scenario was reported [70] where the orientational order of the clusters is crucial.

By interfering five laser beams, a quasicrystalline decagonal substrate can be created [71]. A new pseudomorphic phase was found in real-space experiments of colloids on these quasicrystalline substrates which shows both crystalline and quasicrystalline structural properties. The structural elements of the pseudomorphic phase comprises an archimedean-like tiling with alternating rows of square and triangular tiles. Corresponding theoretical studies reveal a wealth of additional new phases on quasicrystalline substrates, e.g. a quasicrystalline phase with 20 -fold bond order [72]. Finally, the Brownian motion of colloids in quasicrystalline substrate was addressed by simulations revealing a wide-spread subdiffusive regime before ultimate long-time diffusion [73]. 


\subsection{Colloidal particles confined to time-dependent external laser-optical fields}

Clearly, confining particles by a time-dependent environment is important not only for colloids but also for ions in electromagnetic Paul traps [74, 75] and for dust particles in a complex plasma which are confined by external fields [76]. Colloidal particles trapped in time-dependent confinement [77, 78, 79] can serve as model systems to extract and understand the underlying physics. Unlike ions and dust particles in the plasma, however, colloidal systems perform completely overdamped dynamics due to their embedding in a viscous solvent.

While static external confinement potentials lead to equilibrium problems, a timedependent confinement genuinely induces nonequilibrium behaviour and is therefore much more complex and complicated. Time-dependent confinement in colloidal dispersions can be realized best by external laser fields which are externally controllable and can be turned on and off $[77,78,79,5]$. This fascinating possibility has led to recent theoretical studies mainly based on dynamical density functional theory for various space- and time-dependent external potentials $V(\vec{r}, t)$ which we shall briefly describe in the sequel.

The strength of a confinement potential can be suddenly increased or decreased and the relaxation of the system can then be observed. The following time-dependent confining potential was used in [80] in order to describe sudden changes and study the relaxational behaviour of the system:

$$
V(r, t)=\Phi_{0}\left[\left(r / R_{1}\right)^{\alpha} \Theta(-t)+\left(r / R_{2}\right)^{\alpha} \Theta(t)\right]
$$

Here $\Phi_{0}$ is the energy prefactor, and $R_{1}, R_{2}$ are the length scales associated with the range of the confining trap. The exponent $\alpha$ used in (5) models traps of different sharpness, typically $\alpha=2$, but a case of sharp confinement $\alpha=10$ was also examined in [80]. With $\Theta(t)$ denoting the unit step function, this potential is an instantaneous switch of the confinement between two static cases.

A smooth variant in order to continuously interpolate in time between two different static situations was used in [81]

$$
V(z, t)=V_{0}(z / Z(t))^{\alpha}
$$

where $Z(t)$ is a continuous interpolating function. If $Z(t)$ varies slowly on the Brownian time-scale of single particle diffusion, then an adiabatic approximation can be invoked which maps the system on the instantaneous equilibrium potential.

More intricate behaviour is contained in a traveling wave external potential considered in [82]:

$$
V(z, t)=G(z-v t)
$$

Here, $G(x)$ is a shape function and $v$ is the prescribed travel velocity. Here, it is interesting to see whether and how fast colloidal particles can be dragged through the solvent using these traveling wave-like pulling potential. For a finite confinement barrier (i.e. for a bounded function $G(x)$ ), particles are lost for large velocities $v$, and 
the transition between the "dragged" and the "delayed" case deserves more detailed studying.

A globally stable but locally unstable potential was suggested in [83, 84]

$$
V(r, t)=V_{1} r^{4}+V_{2} \cos (\omega t) r^{2}
$$

where $V_{1}$ and $V_{2}$ are positive prefactors and $\omega$ is a driving frequency. This potential tends to infinity or large distances $r$ at any time $t$, therefore it is globally stable implying a localized density profiles at any time. However, close to the origin the potential periodically switches from a stable to an unstable situation. In the steady state, the system will nonlinearly respond periodically in time to this external drive with the same frequency $\omega$ resulting in a periodic expansion and compression. This density oscillation is referred to as breathing mode of the system.

In the next chapter we shall study the extreme case where there is no global stabilizing term. We shall use the simplest form for the potential by setting $V_{1}$ to zero. Then, there is an exploding breathing mode which is governed by a competition between the diffusive Brownian motion and the direct drive of the external potential. At least the initial stage of this process can be realized experimentally by crossed laser beams or by scanning around a single laser tweezer quickly [77, 78, 79, 5].

\section{Giant breathing in potentials switching periodically from stable to unstable situations}

\subsection{Harmonic potentials}

We now focus on the dynamics of a colloidal particle in a time-dependent external potential which is periodically switching from a stable to a globally unstable situation. In detail, we study a time-dependent potential in one spatial dimension ( $x$-direction) which oscillates periodically in time from a stable to an unstable situation. If the potential is quadratic in $x$, the corresponding Langevin equation with overdamped Brownian dynamics can be solved analytically. The oscillator with time-dependent frequencies has been exploited in quite different contexts (also with inertia) ranging from the Brownian parametric oscillator $[85,86]$, the study of multiplicative noise (fluctuating frequencies) [87, 88, 89, 90, 91], stochastic resonance [92, 93] and fluctuation squeezing [94] to the efficiency of stochastic heat engines [95] and the motion of classical particles in electromagnetic traps $[96,97]$. Here we apply the solution to unstable trapping and study the corresponding escape dynamics of a completely overdamped colloidal particle.

The trap potential $V(x, t)$ depends both on the one-dimensional spatial coordinate $x$ and time $t$. The completely overdamped Brownian motion of a classical particle in this potential is governed by the stochastic Langevin equation

$$
\gamma \dot{x}+\partial V(x, t) / \partial x=f(t)
$$

where $\gamma$ is a friction coefficient and the dot denotes a time derivative. A harmonic time-dependent potential is given by

$$
V(x, t)=\lambda(t) x^{2} / 2+F(t) x+C(t)
$$


as illustrated in figure 3 where the time-dependent prefactor $\lambda(t)$ and the external timedependent force $F(t)$ and the irrelevant constant $C(t)$ are prescribed and given. On the right hand side of $(9)$, the stochastic forces $f(t)$ are white noise terms with zero mean, $\overline{f(t)}=0$, and variance $\overline{f(t) f\left(t^{\prime}\right)}=2 \gamma k_{B} T \delta\left(t-t^{\prime}\right)$ with a thermal energy $k_{B} T$. The free motion (where $V(x, t)=0$ ) sets a diffusion coefficient of $D_{0}=k_{B} T / \gamma$ with a mean-square displacement $\Delta(t)$ linear in time, $\Delta(t)=2 D_{0} t$.

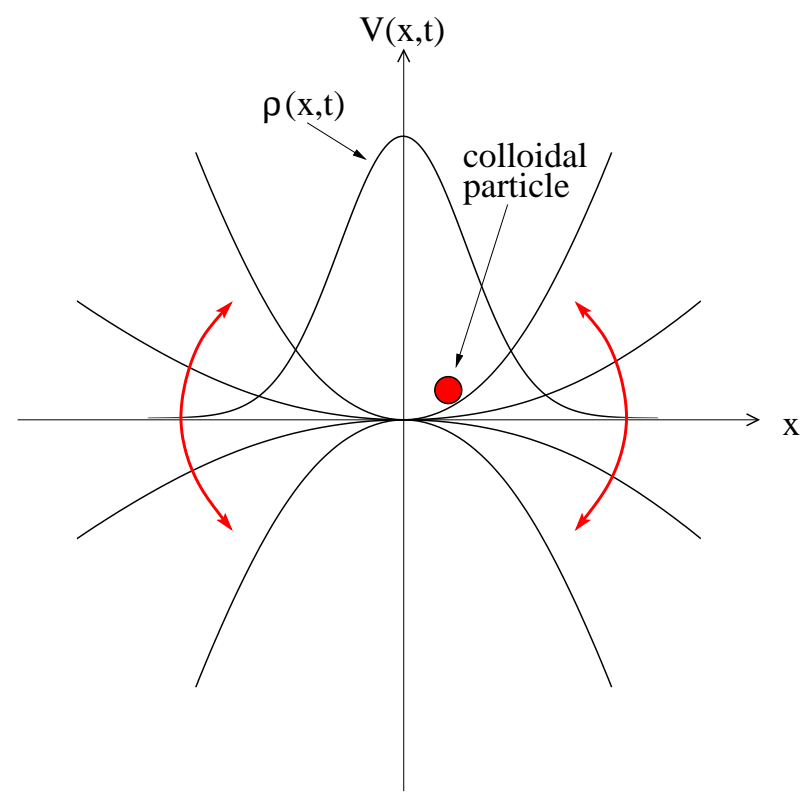

Figure 3. Schematic illustration of the time-dependent potential $V(x, t)$ as a function of the spatial coordinate $x$ for different times $t$. The potential switches periodically in time from a stable to an unstable situation. For simplicity, the symmetric case $F(t)=0$ is shown. The Gaussian one-particle density $\rho(x, t)$ is also indicated.

The analytical solution of (9) with the initial condition $x(t=0)=x_{0}$ is given as

$$
\begin{aligned}
x(t)=\exp ( & \left.-\int_{0}^{t} \mathrm{~d} t^{\prime} \lambda\left(t^{\prime}\right) / \gamma\right) \\
& \times\left[x_{0}+\int_{0}^{t} \mathrm{~d} t^{\prime} \frac{\left(F\left(t^{\prime}\right)+f\left(t^{\prime}\right)\right)}{\gamma} \exp \left(\int_{0}^{t^{\prime}} \mathrm{d} t^{\prime \prime} \lambda\left(t^{\prime \prime}\right) / \gamma\right)\right] .
\end{aligned}
$$

Performing a noise average $\cdots$, we obtain for the mean path

$$
\begin{aligned}
E(t) \equiv \overline{x(t)}= & \exp \left(-\int_{0}^{t} \mathrm{~d} t^{\prime} \lambda\left(t^{\prime}\right) / \gamma\right) x_{0} \\
& +\int_{0}^{t} \mathrm{~d} t^{\prime} \frac{F\left(t^{\prime}\right)}{\gamma} \exp \left(\int_{t}^{t^{\prime}} \mathrm{d} t^{\prime \prime} \lambda\left(t^{\prime \prime}\right) / \gamma\right) .
\end{aligned}
$$

For the mean-square displacement we get

$$
\Delta(t) \equiv \overline{(x(t)-\overline{x(t)})^{2}}=2 D_{0} \int_{0}^{t} \mathrm{~d} t_{1} \exp \left(2 \int_{t}^{t_{1}} \mathrm{~d} t^{\prime} \lambda\left(t^{\prime}\right) / \gamma\right) .
$$


The full solution for the time-dependent one-particle density $\rho(x, t)$ of the Smoluchowski equation

$$
\gamma \frac{\partial \rho}{\partial t}=k_{B} T \frac{\partial^{2} \rho}{\partial x^{2}}+\frac{\partial}{\partial x}\left(\rho \frac{\partial V(x, t)}{\partial x}\right)
$$

which is stochastically equivalent to the Langevin equation (9), is Gaussian and given by

$$
\rho(x, t)=\frac{1}{\sqrt{2 \pi \Delta(t)}} \exp \left(-\frac{(x-E(t))^{2}}{2 \Delta(t)}\right) .
$$

Hence it is completely characterized by its mean $E(t)$ and variance $\Delta(t)$.

Before considering special cases, we calculate the probability $p(t)$ for a particle to go to a negative location after a time $t, x(t)<0$, provided it has been at $x_{0}$ for $t=0$. For $x_{0}>0$, this is the hopping probability to change sign in location. Clearly,

$$
p(t)=\int_{-\infty}^{0} \rho(x, t) \mathrm{d} x=\frac{1}{2} \operatorname{erfc}\left(\frac{E(t)}{\sqrt{2 \Delta(t)}}\right),
$$

where $\operatorname{erfc}(x)=1-\Phi(x)$ is the complementary error function with $\Phi(x)=\frac{2}{\sqrt{\pi}} \int_{0}^{x} \mathrm{e}^{-y^{2}} \mathrm{~d} y$ denoting the probability integral. The transition rate $\Gamma(t)=d p(t) / d t$ to go to the left location is then

$$
\Gamma(t)=-\frac{1}{\sqrt{\pi}} \exp \left(-\frac{E^{2}(t)}{2 \Delta(t)}\right) \frac{d}{d t}\left(\frac{E(t)}{\sqrt{2 \Delta(t)}}\right) .
$$

We now evaluate the behaviour for different cases. The simplest special case is a "seesaw" potential $\lambda(t)=0$ and $F(t)=F_{0} \cos (\omega t+\phi)$, where $F_{0}$ is an amplitude, $\omega$ a frequency and $\phi$ a phase. Here, clearly, $\overline{x(t)}=x_{0}+F_{0} \sin (\omega t+\phi) / \omega \gamma$ and the relative displacement $\Delta(t)=2 D_{0} t$ is identical to the free particle. In this simple reference case, the particle is kicked periodically to the right and left but its diffusion is unaltered with respect to free diffusion. Here $\lim _{t \rightarrow \infty} p(t)=\frac{1}{2}$ and the long-time behaviour of the hopping rate is

$$
\Gamma(t)=-\frac{F_{0} \cos (\omega t+\phi)}{\sqrt{\pi D_{0} 2 \gamma}} \frac{1}{\sqrt{t}}+O\left(\frac{1}{t^{3 / 2}}\right) .
$$

The second special case is a symmetric potential with $F(t)=0$ which periodically switches from a stable to an unstable solution as expressed by

$$
\lambda(t)=\lambda_{0}(a+\Lambda \cos (\omega t+\phi))
$$

The sign of $a$ decides whether the time-averaged potential is stable $(a>0)$ or unstable $(a<0)$. For the parametric oscillator, the case $a>0$ is typically anticipated $[85,86]$. There is a third marginal situation for $a=0$ where the potential has a vanishing timeaverage. Clearly, in this case,

$$
E(t)=x_{0} \exp \left(-\frac{\lambda_{0} a t}{\gamma}-\frac{\lambda_{0} \Lambda \sin (\omega t+\phi)}{\gamma \omega}\right)
$$


and

$$
\begin{aligned}
& \Delta(t)=2 D_{0} \exp \left(-\frac{2 \lambda_{0} a t}{\gamma}-\frac{2 \lambda_{0} \Lambda \sin (\omega t+\phi)}{\gamma \omega}\right) \\
& \times \int_{0}^{t} \mathrm{~d} t^{\prime} \exp \left(\frac{2 \lambda_{0} a t^{\prime}}{\gamma}+\frac{2 \lambda_{0} \Lambda \sin \left(\omega t^{\prime}+\phi\right)}{\gamma \omega}\right) .
\end{aligned}
$$

Let us now discuss the long-time behaviour of (21): For $a>0, \lim _{t \rightarrow \infty} \Delta(t)$ is finite leading to an averaged bounded particle while for $a<0, \lim _{t \rightarrow \infty} \Delta(t)=\infty$. In detail, $\Delta(t)$ diverges exponentially in time but has a superimposed oscillatory part in time as well. We call this unstable oscillatory behaviour giant breathing, since it the amplitude is dramatically amplified as a function of time. The marginal case $a=0$, leads to an oscillating breathing as well, but the amplitude are diverging linearly in time. The corresponding prefactors, however, are different from free particle diffusion.

The behaviour is summarized in figure 4 for $a \in\{1,-1,0\}, \Lambda=1, \omega \tau=1$, and $\phi=0$ where the length, time and energy units are given in terms of $\sigma=\sqrt{k_{B} T / \lambda_{0}}$, $\tau=\sigma^{2} / D_{0}$ and $k_{B} T$, respectively. The linear plot in figure 4a shows the enhancement in the marginal case $a=0$ by comparing it to the free particle diffusion where $\Delta(t) / \sigma^{2}=2 t / \tau$. The diffusive characteristics in this case is best seen on the double logarithmic plot of figure $4 \mathrm{~b}$ where the slope of 1 is clearly emerging. On the other hand, the giant breathing effects for $a<0$ shows up by a slope which increases in time, see again figure $4 \mathrm{~b}$.

The long-time behaviour of $p$ is

$$
\lim _{t \rightarrow \infty} p(t)= \begin{cases}\frac{1}{2} & \text { for } a \geq 0 \\ \frac{1}{2} \operatorname{erfc} X & \text { for } a<0\end{cases}
$$

where

$$
X=\frac{x_{0}}{2 \sqrt{D_{0}}}\left(\int_{0}^{\infty} \mathrm{d} t^{\prime} \exp \left(-\frac{|a| \lambda_{0} t^{\prime}}{\gamma}+\frac{\lambda_{0} \Lambda \sin \left(\omega t^{\prime}+\phi\right)}{\gamma \omega}\right)\right) .
$$

The asymptotic $(t \rightarrow \infty)$ behaviour of the rate $\Gamma(t)$ is proportional to $t^{-3 / 2}$ for $a=0$ as for free diffusion. In detail, the leading order is

$$
\Gamma(t)=\frac{1}{\sqrt{4 \pi}}\left(\frac{x_{0}^{2}}{4 D_{0}}\right)^{1 / 2} \frac{1}{B^{3 / 2}} \exp \left(\frac{2 \lambda_{0} \Lambda \sin (\omega t+\phi)}{\gamma \omega}\right) \frac{1}{t^{3 / 2}}
$$

with $B=\frac{\omega}{2 \pi} \int_{0}^{\frac{2 \pi}{\omega}} \mathrm{d} t \exp \left(\frac{2 \lambda_{0} \Lambda \sin (\omega t+\phi)}{\omega \gamma}\right)$, which is a faster decay than for the seesaw potential (18). For $a \neq 0, \Gamma(t)$ decays exponentially to zero. In detail, for $a>0$, the asymptotically leading term is proportional to

$$
\Gamma(t) \sim \exp \left(-\frac{\lambda_{0} a t}{\gamma}+\frac{2 \lambda_{0} \Lambda \sin (\omega t+\phi)}{\gamma \omega}\right)
$$

while for $a<0$ the leading order is

$$
\Gamma(t) \sim \frac{1}{\sqrt{4 \pi}} \exp \left(-X^{2}\right) X^{3} \frac{4 D_{0}}{x_{0}^{2}} \exp \left(-\frac{2 \lambda_{0}|a| t}{\gamma}+\frac{2 \lambda_{0} \Lambda \sin (\omega t+\phi)}{\gamma \omega}\right) .
$$



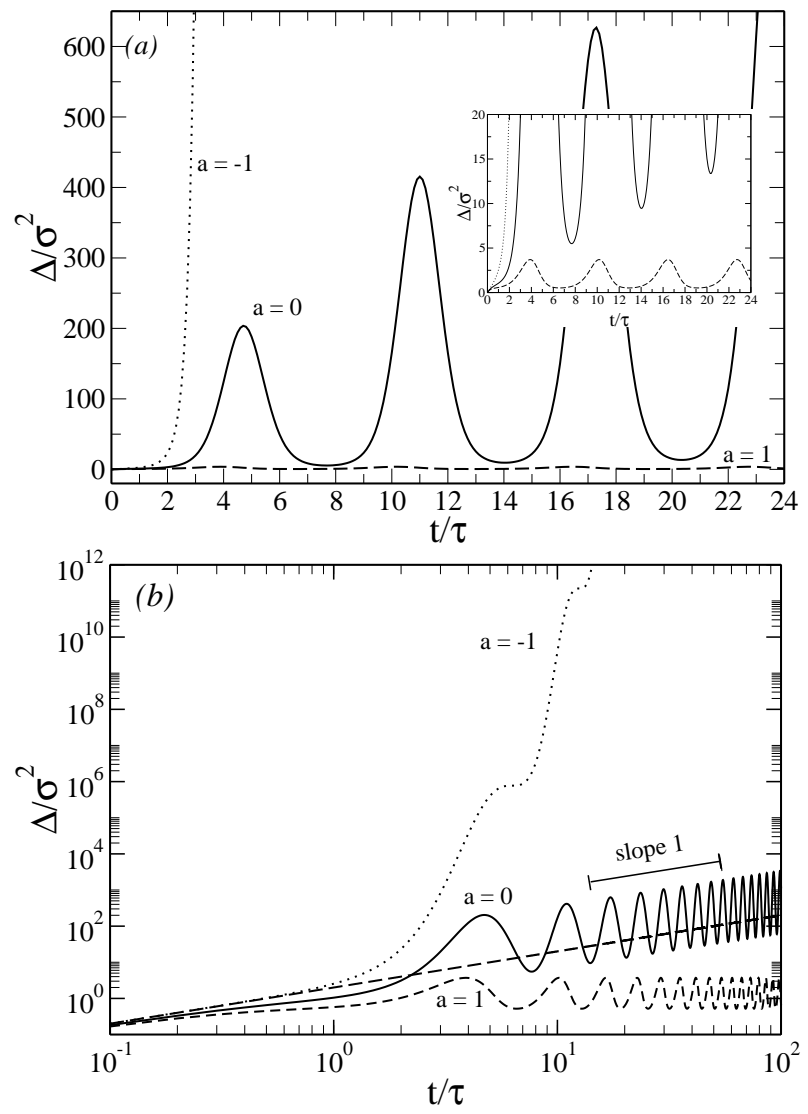

Figure 4. Reduced mean-square displacement $\Delta(t) / \sigma^{2}$ of a particle in an timedependent harmonic trap versus reduced time $t / \tau$ for three different situations: i) $a=1$, i.e. the time-averaged potential is stable, ii) $a=0$, i.e. the time-averaged potential is vanishing, iii) $a=1$, i.e. the time-averaged potential is unstable. a) linear plot, b) double logarithmic plot. The further parameters are $\Lambda=\omega \tau=1$. The dashed line cutting the data for $a=0$ in $\mathrm{b}$ ) denotes free particle diffusion.

For same $|a|$, it decays faster by a factor of 2 in the exponent than in (25), since an unstable potential tends to separate the particle more from zero.

We finally point out that the giant breathing effect is very general. It will certainly occur also in higher spatial dimensions in radially-symmetric potentials where a similar analysis can be performed. Also if a finite number of interacting particles is in the trap, the rapid increase in the mean-square displacement will dominate for long times.

\subsection{Anharmonic potentials}

We next consider nonlinear potentials

$$
V(x, t)=\lambda_{0}(a+\Lambda \cos (\omega t+\phi))|x|^{\alpha} / \alpha
$$

where $\alpha$ is an arbitrary exponent, the special case $\alpha=2$ is leading back to the exactly soluble oscillator. For general $\alpha$ there is no analytical solution available such that one has to resort to a numerical solution of the Langevin equation (9). 


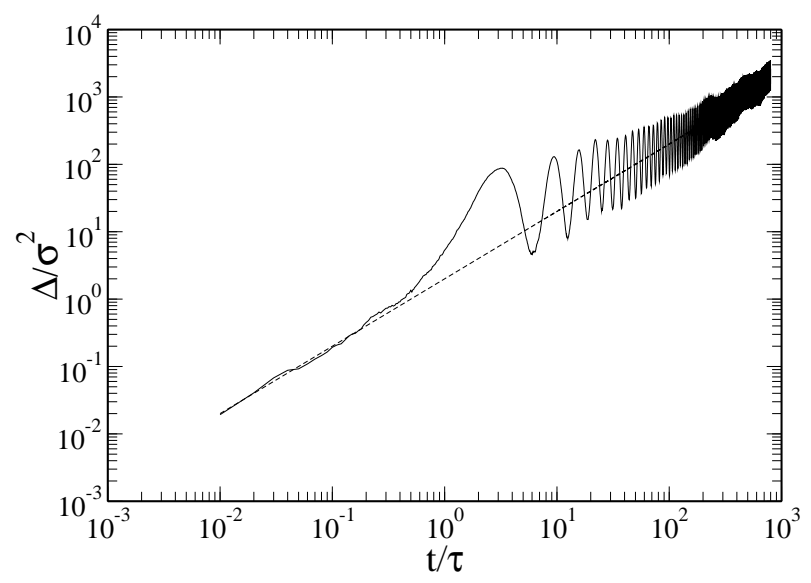

Figure 5. Same as figure $4 \mathrm{~b}$ but now for an anharmonic potential of exponent $\alpha=3 / 2$. The further parameters are $a=0, \omega \tau=1, \phi=-\pi / 2$, and $\Lambda=2$. The dashed line denotes the free particle diffusion.

The length, time and energy units are given in terms of $\sigma=k_{B} T / \lambda_{0}{ }^{1 / \alpha}, \tau=\sigma^{2} / D_{0}$ and $k_{B} T$ respectively. The qualitatively behaviour of the breathing mode is similar to the harmonic case for $0<\alpha<2$. As an example we plot the mean-square displacement for a potential with exponent $\alpha=3 / 2$, on a double-logarithmic scale, see Figure 5 . These data were obtained from a standard Brownian dynamics simulation by solving the Langevin equation (9) numerically. The parameters are $a=0$ corresponding to the marginal case where the time-average of the external potential is vanishing, and $\omega \tau=1$, $\phi=-\pi / 2, \Lambda=2$.

There is short-time diffusive behaviour seen in figure 5. As a reference the free particle diffusion is also shown where $\Delta(t)=2 D_{0} t$, see the dashed line. For longer-times there are significant oscillations but the overall behaviour is consistent with diffusion which is close to the free diffusion. This is qualitatively similar to the marginal harmonic case. On average, the deviations from the free-particle diffusion is, however, weaker here, is that the potential is weaker for larger distances.

Finally when the exponent $\alpha$ is larger than 2, an analysis of the noise-free equation of motion shows that the particle needs a finite time to escape to infinity if the potential is static and unstable. This implies that for $a<0$ and finite $\omega$ the mean-square displacement is infinity.

It would be interesting to prepare different shapes of the trapping potential in an experiment and to check the predictions of the breathing effect.

\section{Conclusions}

In conclusion, the physics of confined colloidal dispersion in external fields [98] has significantly advanced over the last twenty years with important developments in confinement-induced freezing. In particular, a very strong confinement to two spatial dimensions is realizable and the signature of the Kosterlitz-Thouless-Nelson-Halperin- 
Young scenario could be clearly detected in superparamagnetic colloids confined to a two-dimensional air water interface. Binary mixtures of these suspensions are ideal model system to study the glass transition. Layered crystals with unusual structures were observed and theoretically described for charged suspensions between two charged glass plates. Colloids in optical traps and periodic gratings generated by crossed laser beams are another important playground were new aperiodic phases were found.

Time-dependent traps can be used to trigger a response of the systems towards an oscillating external stimulus. The resulting period breathing mode of the system reveals the underlying competition between Brownian dynamics and confinement. In an unstable situation the amplitude grows exponentially in time. This effect of "giant breathing" should be observable in real-space experiments of colloids in various external traps.

\section{Acknowledgments}

I thank T Palberg, A Sengupta, E C Oğuz, S U Egelhaaf, M Jenkins, R Messina and P Hänggi for helpful discussions. This work is supported by the DFG within SFB TR6 (project D3). This paper is dedicated to Richard Palmer, one of the "giants" of the Journal of Physics: Condensed Matter, on the occasion of his retirement from service for the journal.

\section{References}

[1] Dietrich S and Haase A 1995 Phys. Reports 2601

[2] 1989 Molecular Dynamics in Restricted Geometries ed J Klafter and J M Drake (New York: John Wiley)

[3] Strandburg K J 1988 Rev. Mod. Phys. 60161

[4] Löwen H 2001 J. Phys.: Condens. Matter 13 R415

[5] Jenkins M C and Egelhaaf S U 2008 J. Phys.: Condens. Matter 20404220

[6] Pieranski P, Strzelecki L and Pansu B 1983 Phys. Rev.Lett. 50900

[7] Van Winkle D H and Murray C A 1986 Phys. Rev. A 34562

[8] Murray C A, van Winkle D H and Wenk R A 1990 Phase Transitions 2193

[9] Murray C A 1992 Bond-orientational Order in Condensed Matter Systems ed K J Strandburg (New York: Springer)

[10] Weiss J, Oxtoby D W, Grier D G and Murray C A 1995 J. Chem.Phys. 1031180

[11] Chang E and Hone D 1988 Europhys. Lett. 5635

[12] Allahyarov E, D’Amico I and Löwen H 1999 Phys. Rev. E 903199

[13] Murray C A, Sprenger W O and Wenk R A 1990 Phys. Rev. B 42688

[14] Murray C A and Grier D G 1996 Annu. Rev. Phys. Chem. 47421

[15] Pieranski P, Strzlecki L and B. Pansu 1983 Phys. Rev. Lett. 50900

[16] Chou T and Nelson D R 1993 Phys. Rev. E 484611

[17] Schmidt M and Löwen H 1996 Phys. Rev. Lett. 764552

[18] Schmidt M and Löwen H 1997 Phys. Rev. E 557228

[19] Oğuz E C, Messina R and Löwen H 2008, see http://arxiv.org/abs/0811.4763

[20] Neser S, Bechinger C, Leiderer P and Palberg T 1997 Phys. Rev. Lett. 792348

[21] Ramiro-Manzano F, Bonet E, Rodriguez I and Meseguer F 2007 Phys. Rev. E 76050401

[22] Fortini A and Dijkstra M 2006 J. Phys.: Condens. Matter 18 L371 
[23] Ramiro-Manzano F, Meseguer F, Bonet E and Rodriguez I 2006 Phys. Rev. Lett. 97028304

[24] D'Amico I and Löwen H 1997 Physica A 23725

[25] Hansen J-P and Löwen H 2000 Annual Reviews of Physical Chemistry 51209

[26] Allahyarov E, Löwen H and Trigger S 1998 Phys. Rev. E 575818

[27] Bitzer F, Palberg T, Löwen H, Simon R and Leiderer P 1994 Phys. Rev. E 502821

[28] Messina R and Löwen H 2003 Phys. Rev. Lett. 91146101

[29] Likos C N, Németh Z T and Löwen H 1994 J. Phys.: Condens. Matter 610965

[30] Barreira Fontecha A, Schöpe H J, König H, Palberg T, Messina R and Löwen H 2005 J. Phys.: Condens. Matter 17 S2779

[31] Cohen I, Mason T G and Weitz D A 2004 Phys. Rev. Lett. 93046001

[32] Barreira Fontecha A and Schöpe H J 2008 Phys. Rev. E 77061401

[33] Barreira Fontecha A, Palberg T and Schöpe H J 2007 Phys. Rev. E 76050402

[34] Löwen H, Härtel A, Barreira-Fontecha A, Schöpe H J, Allahyarov E and Palberg T 2008 J. Phys.: Condens. Matter 20404221

[35] Renner C, Löwen H and Barrat J-L 1995 Phys. Rev. E 525091

[36] Németh Z T and Löwen H 1998 J. Phys.: Condens. Matter 106189

[37] Simeonova N B and Kegel W K 2004 Phys. Rev. Lett. 93035701

[38] Fehr T and Löwen H 1995 Phys. Rev. E 524016

[39] Nugent C R, Edmond K V, Patel H N and Weeks E R 2007 Phys. Rev. Lett. 99025702

[40] Zahn K, Méndez-Alcaraz J M and Maret G 1997 Phys. Rev. Lett. 79175

[41] Zahn K and Maret G 1999 Current Opinion in Colloid \& Interface Science 460

[42] Löwen H, Messina R, Hoffmann N, Likos C N, Eisenmann C, Keim P, Gasser U, Maret G, Goldberg $\mathrm{R}$ and Palberg T 2005 J. Phys.: Condens. Matter 17 S3379

[43] Froltsov V A, Blaak R, Likos C N and Löwen H 2003 Phys. Rev. E 68061406

[44] Zahn K, Lenke R and Maret G 1999 Phys. Rev. Lett. 822721

[45] Von Grünberg H H, Keim P, Zahn K and Maret G 2004 Phys. Rev. Lett. 93255703

[46] Keim P, Maret G and von Grünberg H H 2007 Phys. Rev. E 75031402

[47] Eisenmann C, Gasser U, Keim P, Maret G and von Grünberg H H 2005 Phys. Rev. Lett. 95185502

[48] Strictly speaking there are no phonons, as the dynamics of the colloids is completely overdamped. Therefore the spectra are formal corresponding to a force-matrix

[49] Keim P, Maret G, Herz U and von Grünberg H H 2004 Phys. Rev. Lett. 92215504

[50] Eisenmann C, Keim P, Gasser U and Maret G 2004 J. Phys.: Condens. Matter 16 S4095

[51] Froltsov V A, Likos C N, Löwen H, Eisenmann C, Gasser U, Keim P and Maret G 2005 Phys. Rev. E 71031404

[52] Eisenmann C, Gasser U, Keim P and Maret G 2004 Phys. Rev. Lett. 93105702

[53] König H, Hund R, Zahn K and Maret G 2005 Eur. Phys. J. E 18287

[54] Kollmann M, Hund R, Rinn B, Nägele G, Zahn K, König H, Maret G, Klein R and Dhont J K G 2002 Europhys. Lett. $\mathbf{5 8} 919$

[55] Hoffmann N, Ebert F, Likos C N, Löwen H and Maret G 2006 Phys. Rev. Lett. 97078301

[56] Assoud L, Ebert F, Keim P, Messina R, Maret G and Löwen H submitted, see also http://arxiv.org/abs/0811.1498

[57] Dillmann P, Maret G and Keim P 2008 J. Phys. Cond. Matt. 20404216

[58] Ebert F, Keim P and Maret G 2008 Eur. Phys. J. E 26161

[59] Assoud L, Messina R and Löwen H 2007 Europhys. Lett. 8048001

[60] Chowdhury A, Ackerson B J and Clark N A 1985 Phys. Rev. Lett. 55833

[61] Chakrabarti J, Krishnamurthy H R, Sood A K and Sengupta S 1995 Phys. Rev. Lett. 752232

[62] Bechinger C, Brunner M and Leiderer P 2001 Phys. Rev. Lett. 86930

[63] Strepp W, Sengupta S and Nielaba P 2002 Phys. Rev. E 66056109

[64] Götze I O, Brader J M, Schmidt M and Löwen H 2003 Mol. Phys. 1011651

[65] Resnick A 2003 J.of Colloid and Interface Science 26255

[66] Brunner M, Dobnikar J, von Grünberg H H and Bechinger C 2004 Phys. Rev. Lett. 92078301 
[67] Apolinario S W S, Peeters F M and Partoens B 2006 Phys. Rev. E 74031107

[68] Melzer A, Klindworth M and Piel A 2001 Phys. Rev. Lett. 87115002

[69] Brunner M and Bechinger C 2002 Phys. Rev. Lett. 88248302

[70] Reichhardt C and Olson C J 2002 Phys. Rev. Lett. 88248301

[71] Mikhael J, Roth J, Helden L and Bechinger C 2008 Nature 454501

[72] Schmiedeberg M and Stark H 2008 Phys. Rev. Lett. 101218302

[73] Schmiedeberg M, Roth J and Stark H 2007 Eur. Phys. J. 24367

[74] Vedel F 1991 Intern. J. of Mass Spectrometry and Ion Processes 10633

[75] Walz J, Siemers I, Schubert M, Neuhauser W and Blatt R 1993 Europhys. Lett. 21183

[76] Melzer A 2003 Phys. Rev. E 67016411

[77] Lutz C et al 2006 Europhys. Lett. 74719

[78] Henderson S et al 2002 Phys. Rev. Lett. 88088302

[79] Martin S et al 2006 Phys. Rev. Lett. 97248301

[80] Dzubiella J and Likos C N 2003 J. Phys.: Condens. Matter 15 L147

[81] Rex M, Wensink H H and Löwen H 2007 Phys. Rev. E 76021403

[82] Rex M, Likos C N and Löwen H 2005 Phys. Rev. E 72021404

[83] Rex M and Löwen H 2008 Phys. Rev. Lett. 101148302

[84] Rex M and Löwen H 2009 Eur. Phys. J. E 28139

[85] Zerbe C, Jung P and Hänggi P 1994 Phys. Rev. E 493626

[86] Brouard S and Plata J 2001 J. Phys. A: Math. Gen. 3411185

[87] Barzykin A V, Seki K and Shibata F 1998 Phys. Rev. E 576555

[88] Berdichevsky V and Gitterman M 1999 Phys. Rev. E 601494

[89] Gitterman M 2005 Physica A 352309

[90] Calisto H, Mora F and Tirapegui E 2006 Phys. Rev. E 74022102

[91] Mankin R, Laas K, Laas T and Reiter E 2008 Phys. Rev. E 78031120

[92] Gammaitoni L, Hänggi P, Jung P and Marchesoni F 1998 Rev. Mod. Phys. 70223

[93] Barzykin A V and Seki K 1997 Europhys. Lett. 40117

[94] Tashiro T and Morita A 2007 Physica A 377401

[95] Schmiedel T and Seifert U 2008 Europhys. Lett. 8120003

[96] Izmailov A F, Arnold S, Holler S and Myerson A S 1995 Phys. Rev. E 521325

[97] Deng Y, Bechhoefer J and Forde N R 2007 J. Opt. A: Pure Appl. Opt. 9 S256

[98] Löwen H 2008 J. Phys.: Condens. Matter 20404201 